\title{
Quantum Bath Control with Nuclear Spin State Selectivity via Pulse-Adjusted Dynamical Decoupling
}

\author{
J. E. Lang $\oplus_{,}^{1, \dagger}$ D. A. Broadway, ${ }^{2,3, *}$ G. A. L. White, ${ }^{2,3}$ L. T. Hall, ${ }^{2}$ A. Stacey, ${ }^{3,4}$ L. C. L. Hollenberg, ${ }^{2,3}$ \\ T. S. Monteiro, ${ }^{1, \$}$ and J.-P. Tetienne ${ }^{2, \pi}$ \\ ${ }^{1}$ Department of Physics and Astronomy, University College London, Gower Street, London WC1E 6BT, United Kingdom \\ ${ }^{2}$ School of Physics, University of Melbourne, Parkville, Victoria 3010, Australia \\ ${ }^{3}$ Centre for Quantum Computation and Communication Technology, School of Physics, University of Melbourne, \\ Parkville, Victoria 3010, Australia \\ ${ }^{4}$ Melbourne Centre for Nanofabrication, Clayton, Victoria 3168, Australia
}

(Received 23 July 2019; published 22 November 2019)

\begin{abstract}
Dynamical decoupling (DD) is a powerful method for controlling arbitrary open quantum systems. In quantum spin control, DD generally involves a sequence of timed spin flips ( $\pi$ rotations) arranged to either average out or selectively enhance coupling to the environment. Experimentally, errors in the spin flips are inevitably introduced, motivating efforts to optimize error-robust DD. Here we invert this paradigm: by introducing particular control "errors" in standard DD, namely, a small constant deviation from perfect $\pi$ rotations (pulse adjustments), we show we obtain protocols that retain the advantages of DD while introducing the capabilities of quantum state readout and polarization transfer. We exploit this nuclear quantum state selectivity on an ensemble of nitrogen-vacancy centers in diamond to efficiently polarize the ${ }^{13} \mathrm{C}$ quantum bath. The underlying physical mechanism is generic and paves the way to systematic engineering of pulse-adjusted protocols with nuclear state selectivity for quantum control applications.
\end{abstract}

DOI: 10.1103/PhysRevLett.123.210401

Quantum baths of nuclear spins at room temperature typically remain in states close to statistical 50:50 mixtures of spin-up and spin-down, for instance, the relative population imbalance is less than $10^{-5}$ at a magnetic field of $1 \mathrm{~T}$. This very low thermal polarization drastically limits sensitivity and fidelity in many applications ranging from NMR to quantum control using quantum nuclear registers. One solution to this challenge is dynamic nuclear polarization (DNP), the transfer of spin polarization from electron spins to nuclear spins $[1,2]$.

Optically polarized electron spins such as those associated with the nitrogen-vacancy (NV) defect in diamond are particularly interesting for DNP [3-12] owing to the relatively high $(\sim 80 \%)$ electron spin polarization achievable on demand, at room temperature [13]. Transfer of NV electron spin polarization based on tuned cross relaxation has been used to polarize spins external to the diamond substrate $[6,14]$. Another technique for polarization transfer is nuclear spin orientation via electron spin locking (NOVEL), which involves continuous driving of the electron spins at a Hartmann-Hahn $(\mathrm{HH})$ resonance with the target nuclei $[3,7,8,12,15]$. Recently, PulsePol, a DD-type protocol allowing polarization transfer at a rate similar to NOVEL [11] by concatenation of two asymmetric sequences, each made of several electronic spin flips with carefully chosen rotation axes, was proposed.
Here we propose a different approach whereby an asymmetry enabling polarization transfer is encoded in the spin flip itself, by deliberately introducing a flip-angle adjustment $\delta \theta \neq 0$. That is, instead of $\pi$ rotations, one deliberately drives rotations of angle $\pi+\delta \theta$. The underlying physical mechanism, explained by Floquet theory, is generic and is found to retain the advantages of DD [16-20] for decoherence protection (away from resonance) and quantum sensing [21-23] (on resonance). Importantly, the approach offers for the first time nuclear state selectivity in the sensing as it splits the usual electron-nucleus resonance into two distinct resonance points, each corresponding to a different quantum nuclear spin state.

We demonstrate a specific realization based on the commonly used Carr-Purcell-Meiboom-Gill (CPMG) pulse sequence. While the nuclear state selectivity offers new possibilities to a wide range of single-NV quantum sensing applications, including high-fidelity quantum control of weakly coupled nuclear spins, here, we demonstrate its effectiveness in nuclear hyperpolarization. We experimentally implement this protocol, termed PolCPMG, on an ensemble of $\sim 10^{5} \mathrm{NV}$ defects in diamond and demonstrate hyperpolarization of the surrounding bath of ${ }^{13} \mathrm{C}$ nuclear spins as well as real-space imaging of the nuclear polarization map on a scale of tens of $\mu \mathrm{m}$.

We consider a system composed of an electron spin $\vec{S}$ coupled with a nuclear spin $\vec{I}$ under a magnetic field aligned 
(a)

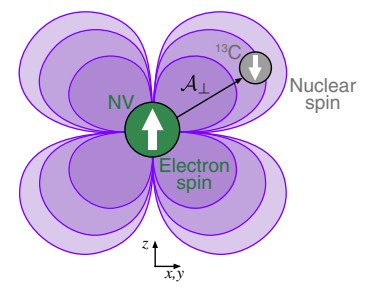

(b)

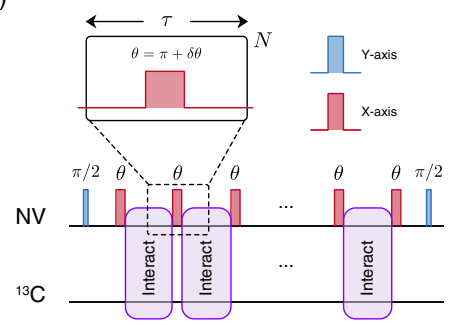

(c)

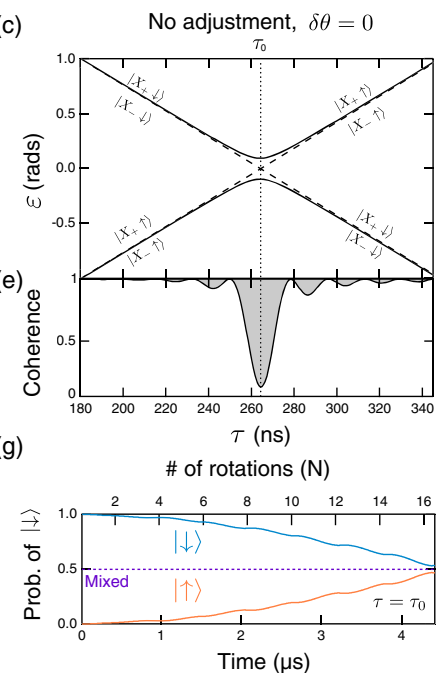

(d)

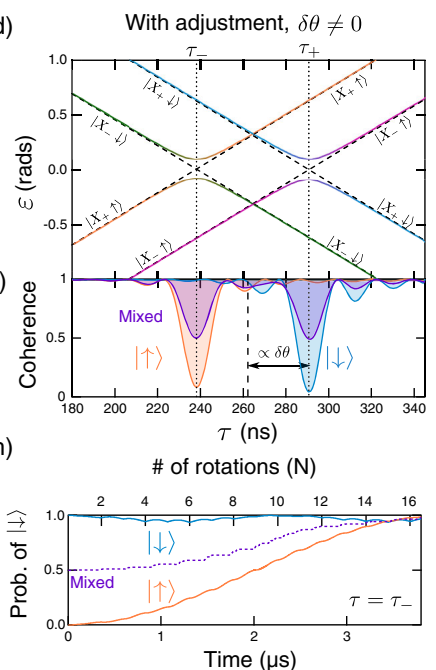

FIG. 1. Concept: adjusted pulses $(\delta \theta \neq 0)$, nuclear state selective resonances and hyperpolarization. (a) Depiction of a central electron spin (e.g., the NV center in diamond) surrounded by a bath of nuclear spins $\left({ }^{13} \mathrm{C}\right)$. The contour lines represent the transverse hyperfine field $\mathcal{A}_{\perp}$, felt by the nuclear spins. (b) Schematic of the PolCPMG dynamical decoupling sequence, which comprises $N$ pulses separated by a period $\tau$. Each pulse rotates the electron spin around the $x$ axis by an angle $\theta=\pi+\delta \theta$, except for the initial and final pulses that rotate the spin by $\pi / 2$ around the $y$ axis. (c),(d) Calculated Floquet phases $\varepsilon$ of the $\mathrm{NV}-{ }^{13} \mathrm{C}$ coupled system periodically driven by the unit sequence shown in (b), as a function of $\tau$, with $\delta \theta=0$ (c) and $\delta \theta=\pi / 10$ (d). Parameters are $\omega_{L}=1.9 \mathrm{MHz}$ (corresponding to a magnetic field $B_{z}=1765 \mathrm{G}$ ) and $\mathcal{A}_{\perp} / 2 \pi=180 \mathrm{kHz}$, typical of the experiments described later. Dashed lines correspond to the uncoupled case $\left(\mathcal{A}_{\perp}=0\right)$. (e), (f) Coherence of the electron spin as a function of $\tau$ after a CPMG (f) and PolCPMG (g) sequence comprising $N=32$ pulses, with the electron spin initialized in $\left|X_{+}\right\rangle$and the nuclear spin initialized in $|\uparrow\rangle$ (orange), $|\downarrow\rangle$ (blue) or in a completely mixed state (purple). In (e) all the different cases are overlapped and shown in black. (g), (h) Time evolution of the nuclear spin state during the CPMG sequence at $\tau=\tau_{0}(\mathrm{~g})$ and during the PolCPMG sequence at $\tau=\tau_{-}$(h), with the system initialized as in (f). Here we used $\mathcal{A}_{\perp} / 2 \pi=380 \mathrm{kHz}$ giving a full polarization transfer in a relatively small number of pulses $(N=16)$, facilitating the visualization of the pulse-to-pulse evolution.

along the $z$ axis [Fig. 1(a)]. The electron spin is subject to a train of microwave pulses with a period $\tau$, with each pulse inducing a rotation of angle $\theta=\pi+\delta \theta$ around the $x$ axis [Fig. 1(b)]. In practice, the rotation angle may be tuned simply via an appropriate frequency detuning and/or pulse duration. In the frame rotating with the driving microwave field, and neglecting counterrotating terms, the Hamiltonian reads [24]

$$
\hat{H}(t)=\omega_{L} \hat{I}_{z}+\hat{S}_{z} \overrightarrow{\mathcal{A}} \cdot \vec{I}+\hat{H}_{p}(t),
$$

where $\omega_{L}$ is the nuclear Larmor frequency, $\overrightarrow{\mathcal{A}}$ is the hyperfine field felt by the nuclear spin (with a perpendicular projection $A_{\perp}$ relative to the $z$ axis), and $\hat{H}_{p}(t)$ is the pulse control Hamiltonian. As the Hamiltonian is periodic, $\hat{H}(t+\tau)=\hat{H}(t)$, Floquet theory provides the natural framework for analyzing the dynamics [39].

The calculated Floquet eigenphases $\varepsilon$ (see details in Ref. [24]) are plotted as a function of $\tau$ for the case of a standard DD sequence $[\delta \theta=0$, Fig. 1(c)] and for the case where a constant flip-angle adjustment $\delta \theta=\pi / 10$ is introduced [Fig. 1(d)]. For the standard $\delta \theta=0$ case, the Floquet eigenstates correspond (far from crossings) to the two nuclear spin states, $|\uparrow\rangle$ and $|\downarrow\rangle$, and are degenerate with respect to the electron spin state. For $\delta \theta \neq 0$, on the other hand, this degeneracy is lifted to produce four distinct Floquet eigenphases, corresponding to $\left|X_{ \pm}\right\rangle|\uparrow \downarrow\rangle$ with $\left|X_{ \pm}\right\rangle=(|0\rangle \pm|1\rangle) / \sqrt{2}$, where $|0\rangle$ and $|1\rangle$ are the $\hat{S}_{z}$ eigenstates of the electron spin. In both cases, avoided crossings arise from the presence of a nonzero hyperfine coupling, indicating the periods $\tau$ for which the driven electron spin has, on average, a nonzero interaction with the nuclear spin [39]. With $\delta \theta=0$, there is a single (degenerate) avoided crossing at $\tau_{0}=\left(\pi / \omega_{L}\right)$. This resonance condition is common to many DD sequences including CPMG and XY8, and is routinely used for sensing nuclear spins $[21,23]$. In a typical NMR sensing experiment, $\tau$ is scanned while monitoring the coherence of the electron spin, producing a spectrum as shown in Fig. 1(e).

With the flip-angle adjustment [Fig. 1(d)], the two avoided crossings occur at two different periods approximately given by (see Ref. [24] for derivation)

$$
\tau_{ \pm} \approx \tau_{0}\left(1 \pm \frac{\delta \theta}{\pi}\right)
$$

This leads to two resonances in the coherence spectrum [Fig. 1(f)]. Importantly, our analysis reveals that these avoided crossings involve pairs of orthogonal electronnuclear states. For instance, the $\tau_{-}$crossing mixes the states 
$\left|X_{+}\right\rangle|\uparrow\rangle$ and $\left|X_{-}\right\rangle|\downarrow\rangle$. This means that a system initialized in $\left|X_{+}\right\rangle|\uparrow\rangle$ and periodically driven at $\tau=\tau_{-}$will undergo oscillations between these two states. Initialization in $\left|X_{+}\right\rangle$ is naturally done in the CPMG sequence through the initial $\pi / 2$ pulse around the $y$ axis when the electron spin is prepared in $|0\rangle$ [Fig. 1(b)], which means that a DNP effect is obtained simply by introducing an angle adjustment in the $\pi$ pulses and choosing $\tau$ accordingly. We note that the splitting of the resonance was previously seen experimentally in Ref. [40] but the novel spin-state selective nature of the two coherence "dips" was overlooked.

We can compare the time evolution of the nuclear spin under the standard CPMG sequence at $\tau=\tau_{0}$ [Fig. 1(g)] and under the PolCPMG sequence at $\tau=\tau_{-}$[Fig. 1(h)]. While the $|\uparrow\rangle$ and $|\downarrow\rangle$ states evolve symmetrically under CPMG, resulting in no change of net polarization for a mixed state, with PolCPMG the $|\downarrow\rangle$ state remains essentially unchanged whereas the $|\uparrow\rangle$ state monotonically evolves to become $|\downarrow\rangle$. The total time required to achieve full polarization transfer can be obtained [24],

$$
t_{\mathrm{PolCPMG}}=\frac{\pi(\pi \pm \delta \theta)}{A_{\perp} \cos \left(\frac{\delta \theta}{2}\right)} \approx \frac{\pi^{2}}{A_{\perp}}
$$

where the \pm sign corresponds to the $\tau_{ \pm}$resonances. This is similar to PulsePol [11] and just a factor $\pi / 2$ longer than with NOVEL, under ideal conditions. In Ref. [24], we examine the relative robustness of these sequences, including another non-DD pulsed protocol, TOP-DNP [41], which employs small rotations $(\theta \ll \pi)$ leading to a very different mechanism for DNP.

For the experiments, a magnetic field $B_{z}=1765 \mathrm{G}$, along the $\mathrm{NV}$ axis, gives a ${ }^{13} \mathrm{C}$ Larmor frequency $\omega_{L} \approx$ $1.9 \mathrm{MHz}$. The signal from an ensemble of $\sim 10^{5} \mathrm{NV}$ centers is measured to average over multiple ${ }^{13} \mathrm{C}$ bath configurations. $\delta \theta$ is controlled via the duration $t_{p}$ of a rectangular pulse [Fig. 2(a)]. Namely, if $t_{p}^{0}=(\pi / \Omega)=40 \mathrm{~ns}$ is the pulse duration for a $\pi$ rotation, where $\Omega$ is the electronic Rabi frequency, we have

$$
\delta \theta=\pi \frac{t_{p}-t_{p}^{0}}{t_{p}^{0}}
$$

We first polarize the ${ }^{13} \mathrm{C}$ bath by applying $R$ repetitions of PolCPMG with a fixed period $\tau=\tau_{ \pm}$, and then probe the state of the bath via a single application of PolCPMG with a variable $\tau$ [Fig. 2(b)]. Coherence spectra obtained for different values of $t_{p}$, corresponding to an adjustment $\delta \theta$ from $-63^{\circ}$ to $+63^{\circ}$, are shown in Fig. 2(c) for $R=0$ (top) and for $R=500$ at $\tau=\tau_{+}$(middle) or $\tau=\tau_{-}$ (bottom). With no polarization $(R=0)$, the bath is in a mixed state and so two resonances are visible at positions well matched by Eq. (2) [dashed lines in Fig. 2(c), top]. With the polarization steps, only one of the two resonances

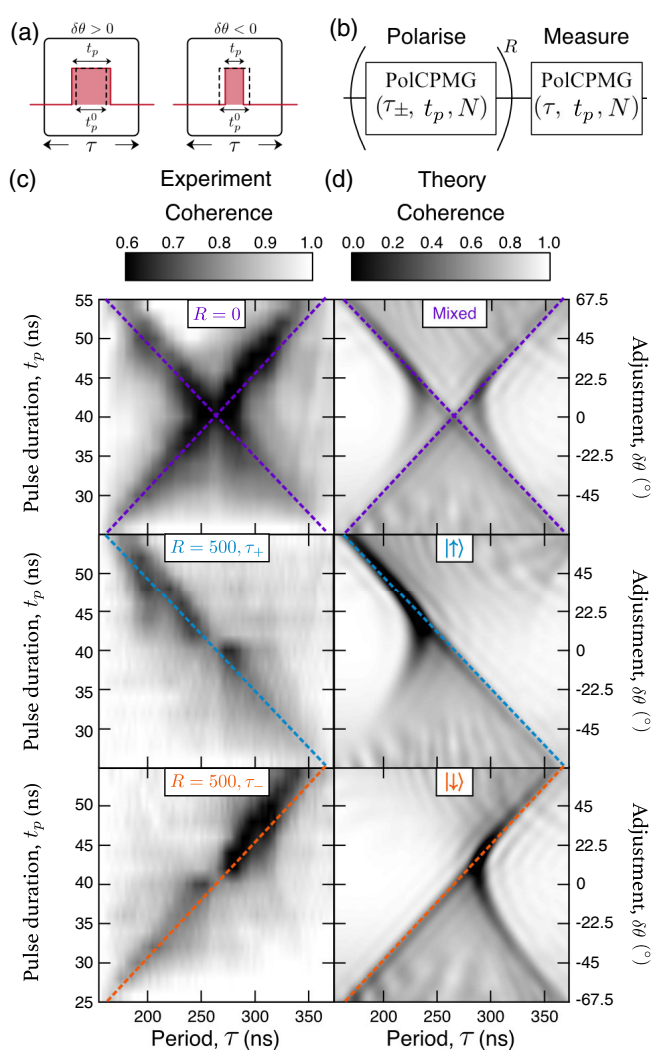

FIG. 2. Observation of ${ }^{13} \mathrm{C}$ hyperpolarization and nuclear state selective addressing. (a) The flip-angle adjustment $\delta \theta$ is controlled by the pulse duration $t_{p}$ relative to the nominal duration $t_{p}^{0}$ corresponding to a $\pi$ rotation. (b) Sequence used to probe the nuclear polarization. (c) NV spin coherence (defined as the probability of finding the NV in $|0\rangle$ after the final $\pi / 2$ pulse) measured with the sequence shown in (b) as a function of $\tau$ and $t_{p}$, with $R=0$ (top plot) and with $R=500$ at $\tau=\tau_{+}$(middle) or $\tau=\tau_{-}$(bottom). Parameters are $\omega_{L} \approx 1.9 \mathrm{MHz}, t_{p}^{0}=40 \mathrm{~ns}$, and $N=32$. (d) Calculated NV spin coherence after a single application of PolCPMG, taking into account the ${ }^{14} \mathrm{~N}$ hyperfine structure and inhomogeneous broadening [24]. The NV is coupled to a single ${ }^{13} \mathrm{C}$ spin $\left(\mathcal{A}_{\perp} / 2 \pi=180 \mathrm{kHz}\right)$ initialized in a mixed state (top plot), in $|\uparrow\rangle$ (middle) and in $|\downarrow\rangle$ (bottom). In (c),(d), the dashed lines correspond to the resonance positions from Eq. (2).

is resolved, indicating that the ${ }^{13} \mathrm{C}$ bath has been efficiently polarized in the $|\uparrow\rangle$ and $|\downarrow\rangle$ states for the $\tau_{+}$and $\tau_{-}$cases [Fig. 2(c), middle and bottom], respectively. Figure 2(c) is a clear demonstration of the nuclear spin state readout enabled by PolCPMG. The different nuclear spin states are addressed independently and present contrasting coherence fingerprints.

The additional features seen in Fig. 2(c) originate mainly from the intrinsic hyperfine splitting due to the nitrogen nucleus of the NV (here ${ }^{14} \mathrm{~N}$, a spin-1). Figure 2(d) shows numerical simulations, including the ${ }^{14} \mathrm{~N}$ nuclear states [24], which correspond to different detunings of the microwave driving frequency, $\Delta \omega$ [24]. With the ${ }^{13} \mathrm{C}$ in a mixed state 

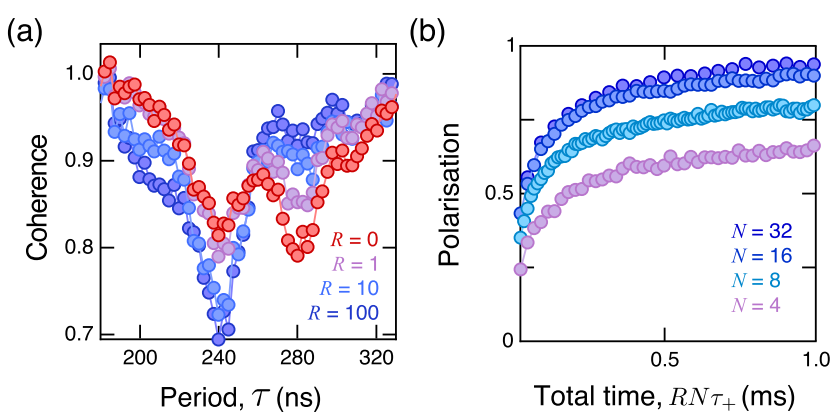

(c)

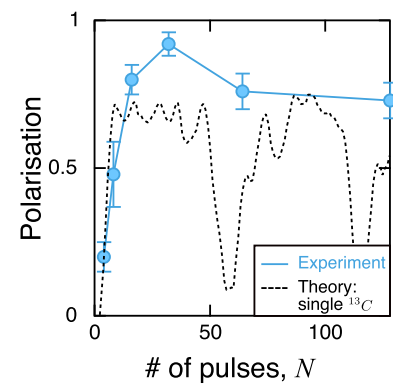

(d)

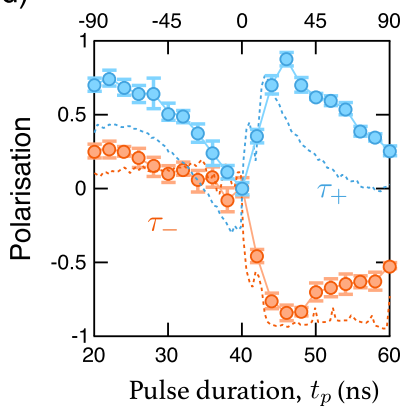

FIG. 3. Polarization dynamics. (a) Coherence spectra taken immediately after $R$ repetitions of the PolCPMG sequence at $\tau=\tau_{+}=288 \mathrm{~ns}$, for different values of $R$, with $N=32$. (b) Polarization of the ${ }^{13} \mathrm{C}$ spin bath as a function of $R$ plotted in terms of the total sequence time, $T=R N \tau_{+}$, for different values of $N$. The polarization is normalized so that a value of +1 $(-1)$ corresponds to all the ${ }^{13} \mathrm{C}$ spins within the NV sensing volume being in the $|\uparrow\rangle(|\downarrow\rangle)$ state. (c) Polarization after $T=$ $1 \mathrm{~ms}$ as a function of $N$. The dashed line is a numerical simulation for a single ${ }^{13} \mathrm{C}\left(\mathcal{A}_{\perp} / 2 \pi=180 \mathrm{kHz}\right)$, including the ${ }^{14} \mathrm{~N}$ hyperfine structure and inhomogeneous broadening. (d) Polarization after $R=500$ cycles as a function of $t_{p}$, with $N=32$. For each value of $t_{p}, \tau$ was adjusted to the resulting $\tau_{+}$ (blue) or $\tau_{-}$(orange) resonance. Dashed lines are numerical simulations.

(top plot), three resonances are resolved near $t_{p}=t_{p}^{0}$, unresolved as a single broad line in the experiment. Interestingly, however, the effect of frequency detunings is largely suppressed in certain regimes, especially for $\delta \theta>0$ (as seen by the larger contrast in Figs. 2(c), 2(d), offering some added robustness, as discussed later.

To study the dynamics of the polarization transfer, we vary the number of repetitions $R$ for a given pulse duration, $t_{p}=44$ ns (i.e., $\delta \theta \approx+18^{\circ}$ ), with the period set to $\tau=\tau_{+}$ polarizing the ${ }^{13} \mathrm{C}$ bath in the $|\uparrow\rangle$ state. Spectra obtained by scanning $\tau$ immediately after the polarization reveal a growth of the $\tau_{-}$resonance and a suppression of the $\tau_{+}$ resonance as $R$ is increased [Fig. 3(a)]. The amplitude of these resonances, $c_{-}$and $c_{+}$, can be used to quantify the ${ }^{13} \mathrm{C}$ polarization, $p=\left(c_{-}-c_{+} / c_{-}+c_{+}\right)$. When all the ${ }^{13} \mathrm{C}$ spins within the sensing volume of the NV probe are polarized in $|\uparrow\rangle(|\downarrow\rangle)$, then $p=+1 \quad(-1)$. Given $A_{\perp} \propto 1 / r^{3}$, the signal is dominated by the nearest spins. For instance, full polarization of the 5 nearest ${ }^{13} \mathrm{C}$ leads to

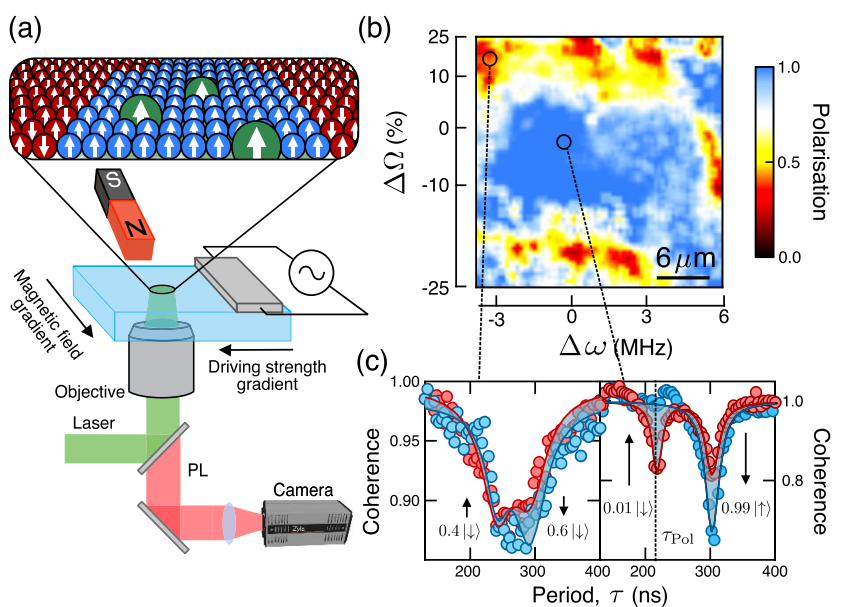

FIG. 4. Imaging nuclear polarization. (a) Experimental setup to image the nuclear polarization. The colored arrows depict the NV spins (green), polarized ${ }^{13} \mathrm{C}$ (blue), and unpolarized ${ }^{13} \mathrm{C}$ (red). (b) Polarization map of a $30 \times 30 \mu \mathrm{m}$ region under deliberately introduced gradients of $\Delta \omega$ along $x$ and of $\Delta \Omega$ along $y$ for $t_{p}=46 \mathrm{~ns}, \tau=\tau_{\mathrm{Pol}}=226 \mathrm{~ns}, N=32, R=400$. (c) PolCPMG spectra from two selected regions with low polarization (left panel) and high polarization (right panel). The dashed vertical line indicates the value of $\tau=\tau_{\mathrm{Pol}}$. In each case, the two curves correspond to the spectrum without polarization $(R=0$, red curve) and with polarization ( $R=400$, blue curve). The lightblue shading highlights the difference between the two curves. Solid lines are two-Lorentzian fits.

$p \approx 0.9$. This local polarization measurement is well suited to the study of the local spin dynamics near isolated NV centers $[7,8,42]$, and complements measurements of the global nuclear polarization obtained by conventional NMR spectroscopy $[4,5,9,10,43]$.

The local polarization $p$ is plotted as a function of the total time $T=R N \tau_{+}$in Fig. 3(b) for different numbers of pulses $(N)$ per cycle, showing a saturation of the polarization after a few $\mathrm{ms}$. We find that for $T>1 \mathrm{~ms}, p$ increases up to $N=32$ before decreasing at larger $N$ [Fig. 3(c)]. For a single ${ }^{13} \mathrm{C}$, this optimum would correspond to a coupling $\mathcal{A}_{\perp} / 2 \pi \approx 180 \mathrm{kHz}$ as deduced from Eq. (3), with a minimum expected at $N=64$ (see dashed line); however, here this should be averaged over multiple $\mathrm{NV}-{ }^{13} \mathrm{C}$ coupling strengths. Using $N=32$, we vary $\delta \theta$ in the range $-90^{\circ} \rightarrow+90^{\circ}$. For each value of $t_{p}$, we apply $R=500$ cycles of the PolCPMG sequence at the corresponding $\tau_{+}$or $\tau_{-}$resonance and then probe the polarization. Figure 3(d) shows that $\delta \theta>0$ yields stronger polarization than $\delta \theta<0$, with maximal $|p|$ at $\sim \delta \theta \approx$ $+30^{\circ}$ for both $\tau_{ \pm}$, an asymmetry also seen in the numerics (dashed lines) and in Fig. 2(d). It originates from the robustness to detuning $\Delta \omega$ of the protocol as a function of $\delta \theta$, and the averaging over the ${ }^{14} \mathrm{~N}$ hyperfine structure and inhomogeneous broadening.

Finally, we demonstrate real-space mapping of the nuclear polarization. We use a wide-field imaging setup 
to illuminate a $1-\mu \mathrm{m}$-thick layer of $\mathrm{NV}$ centers near the diamond surface [Fig. 4(a)] and map the polarization of the ${ }^{13} \mathrm{C}$ bath following application of PolCPMG for $T=1 \mathrm{~ms}$. Gradients of $\Delta \omega$ and of $\Delta \Omega$ were introduced along the $x$ and $y$ spatial direction, respectively [see schematic in Fig. 4(a)]. A polarization image of a $30 \times 30 \mu \mathrm{m}^{2}$ region is shown in Fig. 4(b), revealing a polarization in excess of $80 \%$ in the majority of the image despite a variation of $\Delta \omega=-3.5$ to $6 \mathrm{MHz}$ and of $\Delta \Omega= \pm 25 \%$. As shown in Fig. 4(c), the level of polarization can still be inferred even in far-detuned conditions.

With further improvements, PolCPMG offers a promising route towards the long-standing goal of large-scale NV-based hyperpolarization of external samples (realized recently on single NVs [6-8]), although there remain significant challenges. NV-based hyperpolarization could enable ultrasensitive NMR spectroscopy for in-line chemical analysis or cell biology studies $[44,45]$ or form the basis of a quantum simulator [46] and the ability to directly image the nuclear polarization over tens of $\mu \mathrm{m}$ via nearsurface NVs as demonstrated here may become a ubiquitous tool. More generally, nuclear spin-state selective DD sequences, exemplified by PolCPMG, offer new control possibilities for quantum information processing and quantum sensing.

We acknowledge support from the Australian Research Council (ARC) through Grants No. DE170100129, No. CE170100012, and No. FL130100119. This work was performed in part at the Melbourne Centre for Nanofabrication $(\mathrm{MCN})$ in the Victorian Node of the Australian National Fabrication Facility (ANFF). J. E. L. is funded by an EPSRC Doctoral Prize Fellowship. D. A. B. is supported by an Australian Government Research Training Program Scholarship.

J.E. L. and D. A. B. contributed equally to this work.

†jacob.lang.14@ucl.ac.uk

t.monteiro@ucl.ac.uk

ๆjtetienne@unimelb.edu.au

*david.broadway@unimelb.edu.au

[1] A. Abragam and M. Goldman, Rep. Prog. Phys. 41, 395 (1978).

[2] V. Bajaj, C. Farrar, M. Hornstein, I. Mastovsky, J. Vieregg, J. Bryant, B. Eléna, K. Kreischer, R. Temkin, and R. Griffin, J. Magn. Reson. 160, 85 (2003).

[3] P. London, J. Scheuer, J.-M. Cai, I. Schwarz, A. Retzker, M. B. Plenio, M. Katagiri, T. Teraji, S. Koizumi, J. Isoya, R. Fischer, L. P. McGuinness, B. Naydenov, and F. Jelezko, Phys. Rev. Lett. 111, 067601 (2013).

[4] G. A. Álvarez, C. O. Bretschneider, R. Fischer, P. London, H. Kanda, S. Onoda, J. Isoya, D. Gershoni, and L. Frydman, Nat. Commun. 6, 8456 (2015).
[5] J. P. King, K. Jeong, C. C. Vassiliou, C. S. Shin, R. H. Page, C. E. Avalos, H.-J. Wang, and A. Pines, Nat. Commun. 6, 8965 (2015).

[6] D. A. Broadway, J.-P. Tetienne, A. Stacey, J. D. A. Wood, D. A. Simpson, L. T. Hall, and L. C. L. Hollenberg, Nat. Commun. 9, 1246 (2018).

[7] P. Fernández-Acebal, O. Rosolio, J. Scheuer, C. Müller, S. Müller, S. Schmitt, L. McGuinness, I. Schwarz, Q. Chen, A. Retzker, B. Naydenov, F. Jelezko, and M. Plenio, Nano Lett. 18, 1882 (2018).

[8] F. Shagieva, S. Zaiser, P. Neumann, D. B. R. Dasari, R. Stöhr, A. Denisenko, R. Reuter, C. A. Meriles, and J. Wrachtrup, Nano Lett. 18, 3731 (2018).

[9] D. Pagliero, K. R. K. Rao, P. R. Zangara, S. Dhomkar, H. H. Wong, A. Abril, N. Aslam, A. Parker, J. King, C. E. Avalos, A. Ajoy, J. Wrachtrup, A. Pines, and C. A. Meriles, Phys. Rev. B 97, 024422 (2018).

[10] A. Ajoy, K. Liu, R. Nazaryan, X. Lv, P. R. Zangara, B. Safvati, G. Wang, D. Arnold, G. Li, A. Lin, P. Raghavan, E. Druga, S. Dhomkar, D. Pagliero, J. A. Reimer, D. Suter, C. A. Meriles, and A. Pines, Sci. Adv. 4, eaar5492 (2018).

[11] I. Schwartz, J. Scheuer, B. Tratzmiller, S. Müller, Q. Chen, I. Dhand, Z.-Y. Wang, C. Müller, B. Naydenov, F. Jelezko, and M. B. Plenio, Sci. Adv. 4, eaat8978 (2018).

[12] Y. Hovav, B. Naydenov, F. Jelezko, and N. Bar-Gill, Phys. Rev. Lett. 120, 060405 (2018).

[13] M. W. Doherty, N. B. Manson, P. Delaney, F. Jelezko, J. Wrachtrup, and L. C. L. Hollenberg, Phys. Rep. 528, 1 (2013).

[14] J. D. A. Wood, J.-P. Tetienne, D. A. Broadway, L. T. Hall, D. A. Simpson, A. Stacey, and L. C. L. Hollenberg, Nat. Commun. 8, 15950 (2017).

[15] A. Henstra, P. Dirksen, J. Schmidt, and W. Wenckebach, J. Magn. Reson. 77, 389 (1988).

[16] L. Viola and S. Lloyd, Phys. Rev. A 58, 2733 (1998).

[17] J. J. L. Morton, A. M. Tyryshkin, A. Ardavan, S. C. Benjamin, K. Porfyrakis, S. A. Lyon, and G. A. D. Briggs, Nat. Phys. 2, 40 (2006).

[18] M. J. Biercuk, H. Uys, A. P. VanDevender, N. Shiga, W. M. Itano, and J. J. Bollinger, Nature (London) 458, 996 (2009).

[19] J. Du, X. Rong, N. Zhao, Y. Wang, J. Yang, and R. B. Liu, Nature (London) 461, 1265 (2009).

[20] G. de Lange, Z. H. Wang, D. Ristè, V. V. Dobrovitski, and R. Hanson, Science 330, 60 (2010).

[21] T. Staudacher, F. Shi, S. Pezzagna, J. Meijer, J. Du, C. a. Meriles, F. Reinhard, and J. Wrachtrup, Science 339, 561 (2013).

[22] F. Shi, Q. Zhang, P. Wang, H. Sun, J. Wang, X. Rong, M. Chen, C. Ju, F. Reinhard, H. Chen, J. Wrachtrup, J. Wang, and J. Du, Science 347, 1135 (2015).

[23] I. Lovchinsky, A. O. Sushkov, E. Urbach, N. P. de Leon, S. Choi, K. De Greve, R. Evans, R. Gertner, E. Bersin, C. Müller, L. McGuinness, F. Jelezko, R. L. Walsworth, H. Park, and M. D. Lukin, Science 351, 836 (2016).

[24] See Supplemental Material at http://link.aps.org/ supplemental/10.1103/PhysRevLett.123.210401 for [brief description], which includes Refs. [25-38].

[25] J. E. Lang, J. Casanova, Z.-Y. Wang, M. B. Plenio, and T. S. Monteiro, Phys. Rev. Applied 7, 054009 (2017). 
[26] Z.-H. Wang, G. de Lange, D. Ristè, R. Hanson, and V. V. Dobrovitski, Phys. Rev. B 85, 155204 (2012).

[27] A. Maudsley, J. Magn. Reson. 69, 488 (1986).

[28] T. Gullion, D. B. Baker, and M. S. Conradi, J. Magn. Reson. 89, 479 (1990).

[29] C. A. Ryan, J. S. Hodges, and D. G. Cory, Phys. Rev. Lett. 105, 200402 (2010).

[30] J. H. Shim, I. Niemeyer, J. Zhang, and D. Suter, Europhys. Lett. 99, 40004 (2012).

[31] M. A. Ali Ahmed, G. A. Álvarez, and D. Suter, Phys. Rev. A 87, 042309 (2013).

[32] D. Farfurnik, A. Jarmola, L. M. Pham, Z. H. Wang, V. V. Dobrovitski, R. L. Walsworth, D. Budker, and N. Bar-Gill, Phys. Rev. B 92, 060301(R) (2015).

[33] D. A. Broadway, N. Dontschuk, A. Tsai, S. E. Lillie, C. T.-K. Lew, J. C. McCallum, B. C. Johnson, M. W. Doherty, A. Stacey, L. C. L. Hollenberg, and J.-P. Tetienne, National electronics review 1, 502 (2018).

[34] D. A. Broadway, B. C. Johnson, M. S. J. Barson, S. E. Lillie, N. Dontschuk, D. J. McCloskey, A. Tsai, T. Teraji, D. A. Simpson, A. Stacey, J. C. McCallum, J. E. Bradby, M. W. Doherty, L. C. L. Hollenberg, and J.-P. Tetienne, Nano Lett. 19, 4543 (2019).

[35] D. A. Broadway, J. D. A. Wood, L. T. Hall, A. Stacey, M. Markham, D. A. Simpson, J.-P. Tetienne, and L. C. L. Hollenberg, Phys. Rev. Applied 6, 064001 (2016).

[36] V. Ivády, K. Szász, A. L. Falk, P. V. Klimov, D. J. Christle, E. Janzén, I. A. Abrikosov, D. D. Awschalom, and A. Gali, Phys. Rev. B 92, 115206 (2015).
[37] V. Jacques, P. Neumann, J. Beck, M. Markham, D. Twitchen, J. Meijer, F. Kaiser, G. Balasubramanian, F. Jelezko, and J. Wrachtrup, Phys. Rev. Lett. 102, 057403 (2009).

[38] J. Herrmann, M. A. Appleton, K. Sasaki, Y. Monnai, T. Teraji, K. M. Itoh, and E. Abe, Appl. Phys. Lett. 109, 183111 (2016).

[39] J. E. Lang, R. B. Liu, and T. S. Monteiro, Phys. Rev. X 5, 041016 (2015).

[40] J. E. Lang, T. Madhavan, J.-P. Tetienne, D. A. Broadway, L. T. Hall, T. Teraji, T. S. Monteiro, A. Stacey, and L. C. L. Hollenberg, Phys. Rev. A 99, 012110 (2019).

[41] K. O. Tan, C. Yang, R. T. Weber, G. Mathies, and R. G. Griffin, Sci. Adv. 5, eaav6909 (2019).

[42] D. A. Broadway, S. E. Lillie, N. Dontschuk, A. Stacey, L. T. Hall, J.-P. Tetienne, and L. C. L. Hollenberg, Appl. Phys. Lett. 112, 103103 (2018).

[43] P. R. Zangara, S. Dhomkar, A. Ajoy, K. Liu, R. Nazaryan, D. Pagliero, D. Suter, J.A. Reimer, A. Pines, and C. A. Meriles, Proc. Natl. Acad. Sci. U.S.A. 116, 2512 (2019).

[44] D. B. Bucher, D. R. Glenn, H. Park, M. D. Lukin, and R. L. Walsworth, arXiv:1810.02408.

[45] J. Smits, J. Damron, P. Kehayias, A. F. Mcdowell, N. Mosavian, I. Fescenko, N. Ristoff, A. Laraoui, A. Jarmola, and V. M. Acosta, Sci. Adv. 5, eaaw7895 (2019).

[46] J. Cai, A. Retzker, F. Jelezko, and M. B. Plenio, Nat. Phys. 9, 168 (2013). 